\title{
Transcatheter Closure of Atrial Septal Defect with a CardioSEAL Device
}

\author{
Betau Hwang, MD, Pi-Chang LeE, MD, Yun-Ching Fu, MD, \\ Hsenh-Ping HsING, MD, Sheng-Ling JAN, MD, Pao-Sheng CHIU, MD, \\ Jen-Her Lu, MD, Mei-Yung Jsou, ${ }^{1}$ MD, Zen-Chung WENG, ${ }^{2}$ MD \\ and Laura C.C. MENG, MD
}

\begin{abstract}
SUMMARY
Transcatheter closure of an interatrial septal defect (ASD) with a CardioSEAL device was successfully performed on 12 Taiwanese children. There were five boys and seven girls, aged from 3.6-13.9 $(8.3 \pm 3.2)$ years and with body weight of 15-57 $(33.7 \pm 14.7) \mathrm{kgs}$. After one year of follow-up studies, which included physical examination, ECG, chest X-ray and echocardiography, complete closure of ASD was achieved in nine $(75 \%)$ patients. Two children with a trivial residual shunt were asymptomatic and without audible cardiac murmur. A girl had a small residual left to right atrial shunt by color Doppler echocardiography, but without audible cardiac murmur. There were no immediate or intermediate complications. Transcatheter implantation of the CardioSEAL device is a safe and proper treatment for children with non-complicated small to medium secundum ASD. (Jpn Heart J 2000; 41: 471-480)
\end{abstract}

Key words: Atrial septal defect, Interventional catheterization, Transcatheter closure

INTERATRIAL septal defect (ASD) is one of the most common types of congenital heart disease. ${ }^{1,2)}$ Although it is usually asymptomatic in infancy and childhood, surgery is frequently suggested in the preschool age. ${ }^{1-5)}$ Transcatheter closure with different devices, such as Rashkind umbrella, Sideris' occluder, angel wings device, ASDOS occluder, and Clamshell device have been tried on human beings within the last 20 years. ${ }^{6-15)}$ The initial results were reported as acceptable, but opinions on the best device for transcatheter closure of ASD vary widely. ${ }^{6-17)}$ A total of 12 children were enrolled in the present study to evaluate the immediate and intermediate results of transcatheter closure of ASD by a CardioSEAL device.

From the Department of Pediatrics, ${ }^{1}$ Anesthesiology and ${ }^{2}$ Surgery, Taipei Veterans General Hospital, and National YangMing University, Taipei, Taiwan.

Address for correspondence: BT Hwang, MD, Department of Pediatrics, Taipei Veterans General Hospital, No. 201, Sec II, Shih-Pai Rd, Taipei, Taiwan.

Received for publication December 27, 1999.

Revised and accepted April 28, 2000. 


\section{MATERIALS AND MeTHODS}

A total of 12 Taiwanese children, aged from 3.6 to $13.9(8.3 \pm 3.2)$ years were enrolled in the study. They were five boys and seven girls, with body weight of $15-57(33.7 \pm 14.7) \mathrm{kgs}$. Informed consent was obtained from either of the parents of the 12 patients. The study protocol had been aproved by the Human Ethics Committee of Taipei Veterans General Hospital. All 12 children had undergone physical examination, chest X-ray film, echocardiography (Figure 1) and cardiac catheterization.

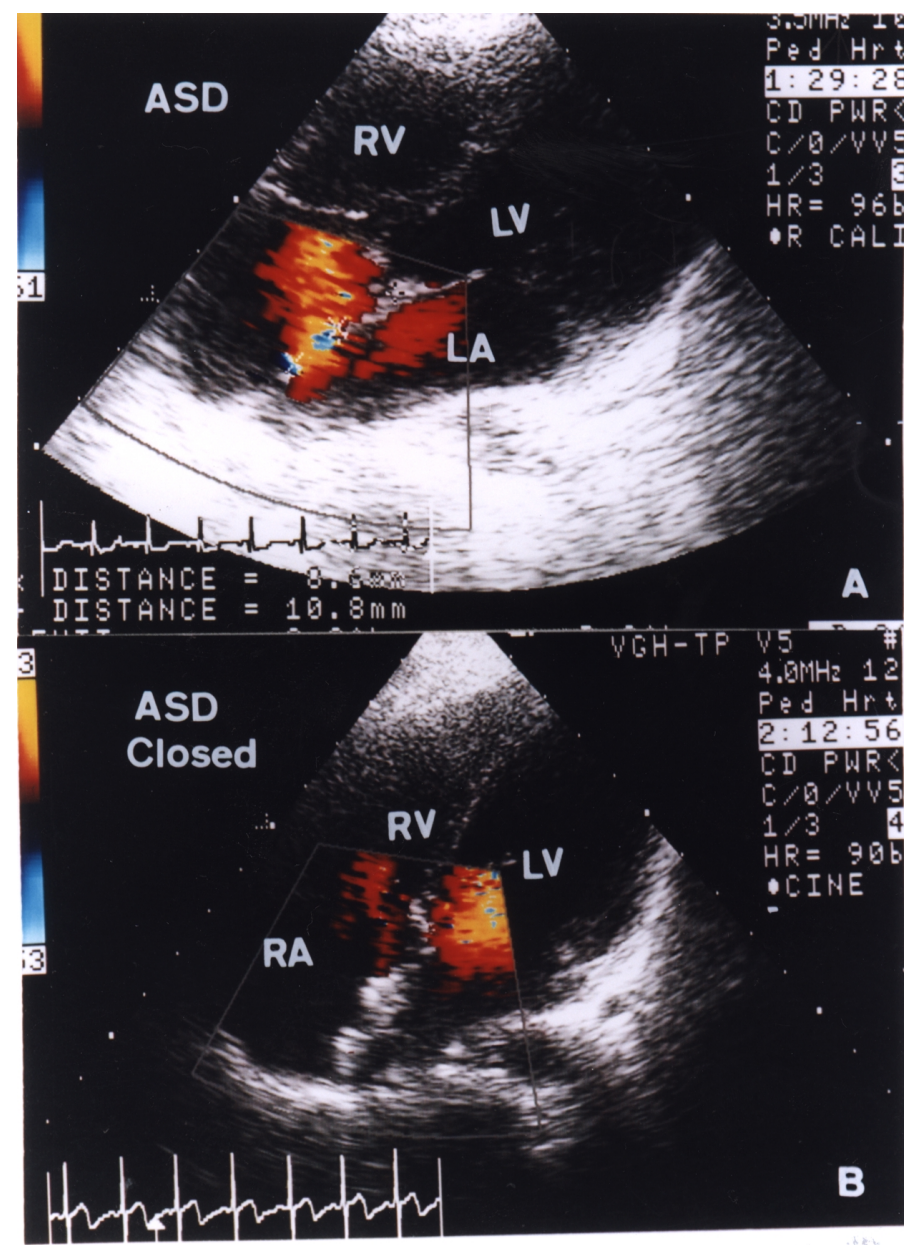

Figure 1. A: Modified subxyphoid view of transthoracic echocardiograms showing a secundum ASD with diameter of $8.6 \mathrm{~mm}$. B: After implantation of CardioSEAL device, the ASD closed completely. ASD = atrial septal defect; RV $=$ right ventricle; $\mathrm{LV}=$ left ventricle; $\mathrm{LA}=$ left atrium. 
The diameter of the ASD was measured by transthoracic (TTE) ${ }^{18-20)}$ and transesophageal echocardiographies (TEE) (Figure 2), ${ }^{21-22)}$ left pulmonary venography and sizing balloon. The rims of the atrial septum around the defect were also measured by TTE and TEE. ${ }^{18-22)}$ Patients were enrolled for evaluation of the possibility of transcatheter closure, if they met the following criteria:

1) aged between 2 and 18 years

2) body weight over $15 \mathrm{~kg}$

3) isolated secundum type ASD



Figure 2. A: Transesophageal echocardiogram showing an ASD. B: After implantation of CardioSEAL device, the ASD closed completely. ASD = atrial septal defect; $\mathrm{LA}=$ left atrium; RA = right atrium. 
4) absence of acute or systemic illness which may influence the course of the procedure

5) maximal diameter of the ASD measured by TTE and TEE $\leqq 15$ $\mathrm{mm}$, and minimal rims surrounding the ASD measured by TTE $\geqq 4$ $\mathrm{mm}$

6) normal adjacent structures surrounding the ASD, without significant mitral or tricuspid regurgitation or stenosis and normal pulmonary venous drainage

7) patients and their guardian are willing to complete the procedure and follow-up studies

8) Qp / Qs ratio calculated according to Fick's principle $\geqq 1.50$ and systolic pulmonary arterial pressure $\leqq 50 \mathrm{mmHg}$

9) Stretched diameter of the ASD measured by sizing balloon $\leqq 20$ $\mathrm{mm}$

\section{PROCEDURES}

The patients underwent standard cardiac catheterization to measure the pressure and $\mathrm{O}_{2}$ saturation in different vessels and cardiac chambers. The Qp / Qs ratio was calculated according to Fick's principle, and pulmonary vascular resistance was calculated by a routine method. After the ASD was identified and measured by pulmonary venography, a 5 or $6 \mathrm{~F}$ end-hole NIH catheter (USCI, Billerica, MA, USA) was put into the upper left pulmonary vein through the ASD. A 0.035 inch guidewire was then inserted to replace the end-hole catheter. The sizing balloon was advanced to the left atrium over the guidewire and then inflated with diluted contrast medium to measure the stretched diameter of the ASD, when it was pulled back to the right atrium. TEE was performed to localize and measure the diameter of the ASD as well as the rims of the atrial septum surrounding the ASD at different directions. ${ }^{19,20)}$ The proper size of the CardioSEAL device (Nitinol Medical Technologies, Boston, USA) was chosen according to the following criteria: 17 or $23 \mathrm{~mm}$ device for ASD with a stretched diameter $\geqq 10 \mathrm{~mm}, 23$ or $28 \mathrm{~mm}$ device for ASD with a stretched diameter of 11-12 mm, 28 or $33 \mathrm{~mm}$ device for ASD with stretched diameter of 13-15 $\mathrm{mm}$, and 33 and $40 \mathrm{~mm}$ device for ASD with a stretched diameter of $16-20 \mathrm{~mm}$.

After the ASD was measured by the sizing balloon, an 11F Mullin sheath (Cook Inc., Bloomington, USA) was inserted into the left atrium over the guidewire. The proper CardioSEAL device was attached to the delivery system and then pushed into the Mullin sheath. Under the guid- 
ance of TEE and fluroscopy, the distal disc of the device was pushed out of the sheath and then opened in the left atrium. The opened disc was adjusted to the proper position under the guidance of TEE. ${ }^{19,20)}$ The Mullin sheath was pulled back to allow the proximal disc to open in the right atrium. After the proper localization of the two discs on both sides of the atrial septum, the discs were separated from the delivery system. Then the Mullin sheath and delivery system were pulled out of the body. Compression of the inguinal region to check bleeding was performed. Antibiotics were given intravenously for two days to prevent possible infection, and $10 \mathrm{mg} / \mathrm{kg}$ of aspirin was given orally for six months to all 12 patients.

All 12 patients successfully underwent the whole procedure and received regular follow-up studies for at least 12 months.

The follow-up studies included complete physical examination, chest X-ray film, ECG, M-mode, as well as two-dimensional Doppler and color Doppler echocardiographies one day, two days, seven days, one month, three months, six months and 12 months after the implantation. ${ }^{22,23,25)}$ The possibilities of arm fracture and disc dislocation were carefully evaluated by echocardiograms and chest X-ray film. ${ }^{23-25)}$ The residual left to right atrial shunt was detected carefully by a color Doppler technique and was graded as trivial, small and moderate according to the color Doppler echocardiographic findings. ${ }^{21,24,25)}$

\section{RESUltS}

The Qp / Qs ratio of these 12 patients ranged from 1.51 to 3.00 $(\mathrm{m} \pm \mathrm{SD}=1.96 \pm 0.53)$. All of the patients studied had normal pulmonary arterial pressure. The average pulmonary arterial pressure was $21.2 \pm 4.0$ / $11.4 \pm 3.7 / 16.0 \pm 3.8 \mathrm{mmHg}$. The calculated pulmonary vascular resistance was $0.3 \sim 0.9(0.5 \pm 0.2)$ Woods units. Ten patients had solitary secun-

\begin{tabular}{|c|c|c|c|}
\hline \multirow{2}{*}{ No. of Patients (12) } & \multirow{2}{*}{$\begin{array}{c}\text { Disappearance of } \\
\text { Cardiac Murmur }(\%)\end{array}$} & \multicolumn{2}{|c|}{$\begin{array}{l}\text { Residual Shunt by } \\
\text { Color Doppler }\end{array}$} \\
\hline & & Small (\%) & Trivial (\%) \\
\hline 1 Day & $11(91.7)$ & $2(16.9)$ & $1(8.3)$ \\
\hline 7 Days & $11(91.7)$ & $2(16.7)$ & $1(8.3)$ \\
\hline 1 Month & $11(91.7)$ & $1(8.3)$ & $2(16.7)$ \\
\hline 3 Months & $11(91.7)$ & $1(8.3)$ & $2(16.7)$ \\
\hline 6 Months & $11(91.7)$ & $1(8.3)$ & $2(16.7)$ \\
\hline 12 Months & $12(100.0)$ & $1(8.3)$ & $2(16.7)$ \\
\hline
\end{tabular}


dum ASD while two patients had two holes visualized by angiographic and TEE findings. One patient who had two separate ASDs needed only one CardioSEAL device to close both holes, while the other one needed two devices to close the separate defects. The diameter of the ASD was $7.6 \pm 2.0(4.0-11.0) \mathrm{mm}$ measured by $2 \mathrm{DE}, 8.5 \pm 3.9(3.0-16.6) \mathrm{mm}$ measured by long axis view of TEE, $5.9 \pm 1.8$ (3.0-8.8) mm measured by short axis view of TEE, $9.5 \pm 2.9(6.3-14.3) \mathrm{mm}$ measured by pulmonary venography, and $10.2 \pm 2.9$ (4.7-15.4) mm measured by stretched balloon.

The size of these 13 CardioSEAL devices was $17 \mathrm{~mm}$ in two, $23 \mathrm{~mm}$ in three and $28 \mathrm{~mm}$ in eight. There were no significant complications after the procedure, except for transient fever noted in three patients, who recovered spontaneously. The follow-up chest X-ray films demonstrated that the arms in all devices remained intact one year after the procedure (Figure 3). The color Doppler echocardiograms showed complete closure of the ASD in nine cases $(75 \%)$ from the second day after implantation of the device. Two cases had color Doppler evidence of small residual left to right atrial shunt, while the other one had only trivial residual shunt with a tiny flow by color Doppler flow mapping from the second day after the procedure (Table).

During one year of follow-up studies, none had any cardiac symptoms. One of two children who initially had a small residual shunt had
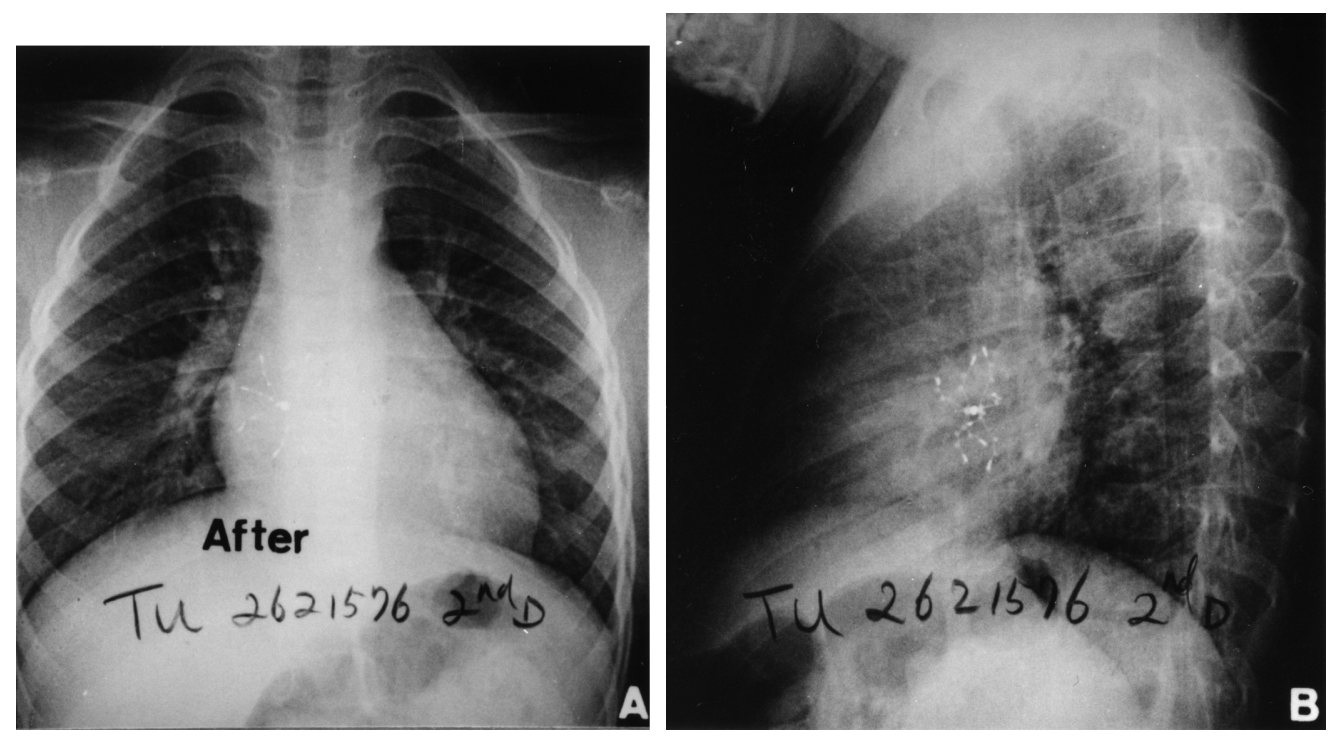

Figure 3. A, B chest X-ray films showing the good position of CardioSEAL device without fracture of arm. 
become only a trivial residual shunt by color Doppler echocardiography. Another patient had a trivial residual shunt which persisted for more than one year. All three paitents were asymptomatic with the absence of audible murmur one year after the procedure (Table).

\section{DISCUSSION}

Since transcatheter closure of ASD was introduced in 1974 by King TD, et al. ${ }^{6}$ many devices have been developed and attempted in human beings. ${ }^{5-15)}$ However, which is the best device for closure of ASD remains controversial. ${ }^{5-17)}$ For central-located secundum ASD of small to medium size, closure with a device is strongly recommended. Sideris' button device had been reported to successfully close secundum ASD in some patients. ${ }^{11-}$ 13,24) Although there was trivial residual leakage in $27 \%$ of patients, ${ }^{20)}$ it has been reported as a safe and effective mothod for closing ASD without surgery. ${ }^{20,24)}$ A clamshell device has also been attempted in many patients, giving encouraging results. But fracture of the arm was reported by radiographic follow-up studies in some patients. ${ }^{23,25)}$ Hence, the CardioSEAL device has been developed based on modification of the Clamshell device. ${ }^{25)}$ The CardioSEAL device maintains the original concept of the Clamshell device. But to lessen the possibility of device arm fractures, an alloy (MP 35N) has been used to construct the support arm to present metal fatigue. Mechanical stress to critical areas of the device arm was also reduced by using two elbow joints on each arm rather than the single joint of the original Clamshell device. $\left.{ }^{25}\right)$

The CardioSEAL device was successfully implanted in the present 12 patients with secundum ASDs of small to medium size. There were no immediate or intermediate complications except for transient fever of about $39^{\circ} \mathrm{C}$ within 24 hours after the procedure in three cases, and local subcutaneous hematoma over the right inguinale region in eight cases. After one year of follow-up studies, all 12 patients were fine without evidence of infection, arrhythmia, congestive heart failure or fracture of the device arm. These findings strongly support the safety of the procedure and this particular device. The complete closure rate of ASD by the CardioSEAL device was $75 \%$ in this series. Two patients had a trivial residual shunt without an audible cardiac murmur indicating the insignificance of the residual shunt. The one patient who had a small residual shunt was also asymptomatic and without audible cardiac murmur. These results were compatible with those of previous reports. ${ }^{22,25)}$ Although an atrial septal defect occlusion system (ASDOS, Sulzer Osypka, Rheinfelden, Germany) 
has been tried in many European countries, ${ }^{16)}$ it has a moderate / larger residual shunt in $2 \%$ and a small residual shunt in $26 \%$ of patients one year after the implantation, ${ }^{16)}$ Furthermore, complications included cerebral thrombosis, late atrial wall perforation, infections in $14 \%$, and frame deformity in $4 \%$ of the patients during the 230 patients-years of followup study. ${ }^{16)}$ Transfemoral approach over both side femoral arteries is also a technical difficulty associated with this particular device. ${ }^{13,16)}$ The buttoned device of Sideris has been tried worldwide to close the ASD. ${ }^{13)}$ The intermediate-term results demonstrated a complete closure rate of $74 \%$ after 224 patient-years of follow-up. Although all the residual shunts were quantitated as being trivial, unbuttoning, late migration and destruction of the mitral valve have been reported. ${ }^{13,24)}$ The device has not been approved by the FDA in the United States. An angel wing device has also been tried since 1993. ${ }^{13)}$ The initial results were encouraging, however, it is available in the United States only experimentally under the FDA protocol. ${ }^{13,14,17)}$ The implant procedure and technique of the CardioSEAL device is not more difficult than that of the ASDOS or Sideris device. The immediate and intermediate results of the CardioSEAL device were similar or better to those of other devices.

The factors influencing the outcome include the proper size and location of ASD, sufficient length of the rims around the defect, especially the anterior-inferior rim, better guidance of transesophageal echocardiography, the technical experience of the pediatric cardiologists and the correct choice of the device. ${ }^{18-22,26)}$ We chose patients with a defect of less than $2.0 \mathrm{~cm}$ in diameter measured by the stretched balloon method in this study. However, we hope it can be applied to the closure of bigger ASDs with the new generation of CardioSEAL STARFlex ${ }^{\mathrm{TM}}$ occluder in the future. Transesophageal echocardiographic assistance is not only the key to identification of the size and location of ASD, but also the best guidance for implantation of the device. ${ }^{21,22)}$ Three-dimensional reconstruction of ASD images by TEE has also been reported as a good reference before transcatheter closure of ASD and for long-term follow up. ${ }^{26)}$

Although children with ASDs are usually asymptomatic, closure of this defect is still strongly recommended in childhood to avoid possible complications in later years. ${ }^{1-5)}$ Surgical correction either by an open heart procedure or by a video-assisted scopic method usually involves some risks. ${ }^{4,5)}$ A surgical scar on the chest is unavoidable. In view of this transcatheter closure of ASD, this device will be the preferred choice for patients with proper size and location of ASD. In addition, a shortening of the hospital stay, avoidance of unnecessary blood transfusions and its 
related complications such as infection, can also be achieved by this method. Hence, transcatheter implantation of the CardioSEAL device is a safe and effective method for closing secundum ASDs of small to medium size. However, the long-term result of this particular device warrants further evaluation. ${ }^{6,7,13)}$

\section{ACKNOWLEDGMENT}

The authors would like to thank the Children's Heart Foundation, R.O.C. and Research Foundation of Cardiovascular Medicine for the financial support, Prof. WE Hellenbrand of Columbia University, NY, USA for his distinguished suggestions, as well as Miss JL Hsing and Miss YY Weng for their assistance in preparing this manuscript.

\section{REFERENCES}

1. Campbell M. Natural history of atrial septal defect. Br Heart J. 1970; 32: 820-6.

2. Campbell M, Neill C, Suzman S. The prognosis of atrial septal defect. BMJ 1957; 1: 1375-83.

3. Craig RJ, and Selzer A. Natural history and prognosis of atrial septal defect. Circulation 1968; 805-15.

4. Galal MO, Wobst A, Halees Z, et al. Peri-operative complications following surgical closure of atrial septal defect type II in 232 patients-a baseline study. Curop H J 1994; 15: 1381-4.

5. Pastork JS, Allen HD and Davis JT. Current outcomes of surgical closure of secundum atrial septal defect. Am J Cardiol 1994; 74: 75-7

6. King TD, Mills NL. Nonoperative closure of atrial septal defects. Surgery 1974; 75: 383-8.

7. King TD, Thompson SL, Steiner C, Mills NL. Secundum atrial septal defect: Nonoperative closure during cardiac catheterization. JAMA 1976; 235: 2506-9.

8. Boutin C, Musewe NN, Smallhorn JF, Dyck JD, Kobayashi T, Benson LN. Echocardiographic follow-up of atrial septal defect after catheter closure by double-umbrella device. Circulation 1993; 88: 621-7.

9. Lloyd TR, Ran PS, Beekman RH 3rd, Medelsohn AM, Sideris EB. Atrial septal defect occlusion with the buttoned device (a multi-institutional U.S. trial). Am J Cardiol 1994; 73: 286-91.

10. Lock JE, Cockerham JT, Keane JF, Finley JP, Wakely PE, Fellows KE. Transcatheter umbrella closure of congenital heart defects. Circulation 1987; 75: 593-9.

11. Rao PS, Wilson AD, Levy JM, Gupta VK, Chopra PS. Role of "buttoned" double-disc device in the management of atrial septal defects. Am Heart J 1992; 123: 191-200.

12. Sideris EB, Sideris SE, Thanopoulos BD, Ehly RL, Fowlkes JP. Transvenous atrial septal defect occlusion by the buttoned device. Am J Cardiol 1990; 66: 1524-6.

13. Latson LA. Pre-Catheter ASD closure. Ped Cardiol. 1998; 19: 86-93.

14. Das GS, Voss G, Jarvis G, et al. Experimental atrial septal defect closure with a new transcatheter self-centering device. Circulation 1993; 88 (part 1): 1754-64.

15. Rickers C, Hamm C, Stern H, et al. Percutaneous closure of secundum artial septal defect with a new self centering device (angel wings). Heart 1998; 80: 517-21.

16. Sievert H, Babic UU, Hausdorf G, et al. Transcatheter closure of atrial septal defect and patent foramen ovale with the ASDOS device (A multi-institutional European trial). Am J Cardiol 1998; 82: 1405-13.

17. Das GS, Shrivastava S, Olaughlin MP, et al. Intermediate term follow-up of patients after percutancous closure of atrial septal defects with the DAS Angel Wings device. Circulation 1996; 94: I-54.

18. Rao PS, Langhough R, Beekman RH, Lloyd TR and Sideris EB. Echocardiographic estimation of balloonstretched diameter of secundum atrial septal defect for transcatheter occlusion. Am H J 1992; 124: 172-5. 
19. Rosenfeld HM, Van Der Velde ME, Sanders SP, et al. Echocardiographic predictors of candidacy for successful transcatheter atrial septal defect closure. Cathet Cardiovasc Diagn 1995; 34: 29-34.

20. Reddy SC, Rao PS, Ewenko J, Koscik R, Wilson AD. Echocardiographic predictors of success of catheter closure of atrial septal defect with the buttoned device. Am H J 1995; 129: 76-82.

21. Ishii M, Kato H, Takagi J, et al. Biplane transesophageal echo-Doppler studies of atrial septal defects: Quantative evaluation and monitoring for transcatheter closure. Am H J 1993; 125: 1363-8.

22. Hellenbrand WE, Fahey JT, McGowan FX, Weltin GG, Kleinman CS. Transesophageal echocardiographic guidance of transcatheter closure of atrial septal defect. Am J Cardiol 1990; 66: 207-13.

23. Schlesinger AE, Folz SJ, Beekman RH. Transcatheter atrial septal defect occlusion devices: normal radiographic appearances and complications. J Vasc Intervent Radiol. 1992; 3: 527-33.

24. Zamora R, Rao PS, Lloyd TR, Beekman RH $3^{\text {rd }}$, and Sideris EB. Intermediate-term results of Phase I Food and Drug Adminstration trials of buttoned device occlusion of secundum strial septal defects. JACC 1998; 31: 6744.

25. Boutin C, Musewe NN, Smallhorn JF, Dyck JD, Kobayashi and Benson LN. Echocardiographic follow-up of atrial septal defect after catheter closure by double-umbrella device. Circulation 1993; 88: 621-7.

26. Lu JH, Hsu TL, Hwang BT, Weng ZC. Visualization of secundum atrial septal defect using transthoracic threedimensional echocardiography in children. Echocardiography 1998; 15: 651-9. 